\title{
A REMARK ON FREE TOPOLOGICAL GROUPS WITH NO SMALL SUBGROUPS
}

\author{
H. B. THOMPSON
}

(Received 2 April 1973; revised 27 July 1973)

Communicated by G. Szekeres

\section{Introduction}

For a completely regular space $X$ let $G(X)$ be the Graev free topological group on $X$. While proving $G(X)$ exists for completely regular spaces $X$, Graev showed that every pseudo-metric on $X$ can be extended to a two-sided invariant pseudo-metric on the abstract group $G(X)$. The free group topology on $G(X)$ is usually strictly finer than this pseudo-metric topology. In particular this is the case when $X$ is not totally disconnected (see Morris and Thompson [7]). It is of interest to know when $G(X)$ has no small subgroups (see Morris [5]). Morris and Thompson [6] showed that this is the case if and only if $X$ admits a continuous metric. The proof relied on properties of the free group topology and it is natural to ask if $G(X)$ with its pseudo-metric topology has no small subgroups when and only when $X$ admits a continuous metric. We show that this is the case. Topological properties of $G(X)$ associated with the pseudo-metric topology have recently been studied by Joiner [3] and Abels [1].

\section{Notation and preliminaries}

Let $X$ be a completely regular topological space with a distinguished point $e$.

The space $X$ is said to admit a continuous metric if there is a continuous one-to-one mapping of $X$ onto a metric space.

A topological group is said to have no small subgroups if there exists a neighbourhood of the identity which contains no non-trivial subgroups.

As the proof of our result depends on Graev's extension of pseudo-metrics on $X$ to $G(X)$ we describe the essential features of this process.

The group $G(X)$ is said to be the Graev topological group on $X$ [2] if it has the properties:

(a) $X$ is a subspace of $G(X)$, 
(b) $X$ generates $G(X)$ algebraically, and $e$ is the identity element of $G(X)$,

(c) for any continuous mapping $\phi$ of $X$ into any topological group $H$ such that $\phi(e)$ is the identity element of $H$, there exists a continuous homomorphism $\Phi$ of $G(X)$ into $H$ such that $\Phi \mid X=\phi$.

Graev showed that $G(X)$ is the free abstract group on the set $X-e$ having the finest group topology which induces the given topology on $X$. Let $X^{\prime}=X \cup\left\{x^{-1}: x \in X-e\right\}$ and $N=\{1,2, \cdots\}$. For $w$ in $G(X)$ with reduced form $w=x_{1} \cdots x_{n}$ let $S(w)=\left\{x_{1}, \cdots, x_{n}, e, x_{1}^{-1}, \cdots, x_{n}^{-1}\right\}$. The topology on $X$ is defined by a family of continuous pseudo-metrics. Let $\rho$ be a pseudo-metric on $X$. Graev extended $\rho$ to a two-sided invariant pseudo-metric on $G(X)$ as follows. Extend $\rho$ to $X^{\prime}$ by setting $\rho\left(x^{-1}, y^{-1}\right)=\rho(x, y)$ and $\rho\left(x^{-1}, y\right)=\rho(x, e)+\rho(e, y)$ for $x$ and $y$ in $X$. For $u$ and $v$ in $G(X)$ we have an infinity of representations $u=x_{1} \cdots x_{n}$ and $v=y_{1} \cdots y_{n}$ where the $x_{i}$ and $y_{i}$ are in $X^{\prime}$. Extend $\rho$ to $G(X)$ by setting

$$
\rho(u, v)=\inf \left\{\sum_{i=1}^{n} \rho\left(x_{i}, y_{i}\right): u=x_{1} \cdots x_{n} \text { and } v=y_{1} \cdots y_{n}\right\}
$$

We are interested in the case $v=e$ and Graev's results restricted to this case are that the infimum is attained when $u$ has its reduced representation $x_{1} \cdots x_{n}$ and the $y_{i}$ are suitably chosen from $S(u)$.

We need the following result (see Kurosh [4], page 127).

LeMma 1. For any $w \in G(X)-e$ there is $l \in G(X)$ and $c \in G(X)-e$ such that $w=l c l^{-1}$ where $c$ has the reduced form $c=x_{1} \cdots x_{n}$ where $x_{i} \in X^{\prime}-e$ for $i=1, \cdots, n$ for some $n \in N$ and $x_{1} \neq x_{n}^{-1}$. Further for any $t \in N, l^{-1} w^{t} l=c^{t}$ and $c^{t}$ has reduced form $c^{t}=x_{1} \cdots x_{n} x_{1} \cdots x_{n} \cdots x_{1} \cdots x_{n}$.

Let $X$ admit a continuous metric $d$. Extend $d$ to a continuous pseudo-metric on $G(X)$ as described above. For any $w$ in $G(X)-e$ set

$$
f(w)=\min \{d(p, q): p \neq q ; p, q \in S(w)\}
$$

The following properties of $f$ need no explanation.

LEMMA 2. The function $f$ satisfies

(i) $f(w)>0$ for all $w \in G(X)-e$,

(ii) if $w$ has reduced form $l \mathrm{~cm}$ for some $l, c$, and $m \in G(X)-e$, then $f(c) \geqq f(w)$, and

(iii) for any $c \in G(X)-e$ and any $t \in N, f\left(c^{t}\right)=f(c)$.

\section{Results}

Let $c$ in $G(X)$ have the reduced form $c=x_{1} \cdots x_{n}$ where the $x_{i}$ are in $X^{\prime}$ ind $x_{1} \neq x_{n}^{-1}$. 
LEMMA 3. If $n \geqq 3$, then for any $t \in N$

$$
d\left(c^{t}, e\right) \geqq t f(c) \text {. }
$$

PRoof. In reduced form $c^{t}=x_{1} \cdots x_{n} x_{1} \cdots x_{n} \cdots x_{1} \cdots x_{n}=s_{1} \cdots s_{t n}$ where $s_{i}=x_{j}$ for $i=(p-1) n+j, 1 \leqq p \leqq t$ and $1 \leqq j \leqq n$. From Graev's construction described in the previous section we may write $e=y_{1} \cdots y_{t n}$ such that $d\left(c^{t}, e\right)=\sum_{i=1}^{t n} d\left(s_{i}, y_{i}\right)$ where $y_{i} \in S\left(c^{t}\right)=S(c)$. It suffices to show that for each $p$ with $1 \leqq p \leqq t, \quad \sum_{i=(p-1) n+1}^{(p-1) n+3} d\left(s_{i}, y_{i}\right) \geqq f(c)$. Consider the case $p=1$ where the inequality becomes

$$
d\left(x_{1}, y_{1}\right)+d\left(x_{2}, y_{2}\right)+d\left(x_{3}, y_{3}\right) \geqq f(c) .
$$

Now $y_{2} \in S(c)$ and if $y_{2} \neq x_{2}$ then $d\left(x_{2}, y_{2}\right) \geqq f(c)$ and the result follows. If $y_{2}=x_{2}$, since $y_{1} \cdots y_{t n}=e$, either $y_{1}=x_{2}^{-1}$ or $y_{3}=x_{2}^{-1}$ so that either $d\left(x_{1}, y_{1}\right)=d\left(x_{1}, x_{2}^{-1}\right) \geqq f(c)$ or $d\left(x_{3}, y_{3}\right)=d\left(x_{3}, x_{2}^{-1}\right) \geqq f(c)$, and the inequality follows. The cases $2 \leqq p \leqq t$ follow analogously.

THEOREM 4. If $X$ admits a continuous metric then $G(X)$ with the pseudometric topology has no small subgroups.

REMARK. If $G(X)$ has no small subgroups then $X$ must admit a continuous pseudo-metric, by [6].

Proof. Let $d$ be the extension of the continuous metric on $X$ to a two-sided invariant pseudo-metric on $G(X)$. The open set $U=\{w \in G(X): d(w, e)<1\}$ contains no nontrivial subgroups. This can be seen as follows. Let $w \in U-e$. Then by Lemma $1, w^{3}=1 c 1^{-1}$ where $1 \in G(X)$ and $c=x_{1} \cdots x_{n}, x_{1} \neq x_{n}^{-1}$, and length $c \geqq 3$. Therefore for $t \in N, d\left(w^{3 t}, e\right)=d\left(1 c^{t} 1^{-1}, e\right)=d\left(c^{t}, e\right)$ by two-sided invariance of $d$. By Lemmas 2 and $3,\left(d\left(c^{t}, e\right) \geqq t f(c) \geqq t f(w)\right.$. Thus $d\left(w^{3 t}, e\right) \geqq t f(w)$ and for $t>f(w)^{-1}, w^{3 t} \notin U$. Therefore $U$ cannot contain a nontrivial subgroup.

\section{References}

[1] Herbert Abels, 'Normen auf freien topologischen Gruppen', Math. Z. 129 (1962), 25-42.

[2] M. I. Graev, 'Free topological groups', Izv. Akad, Nauk SSSR Ser. Mat. 12 (1948), 279-324, (Russian). English Transl. Amer. Math. Soc. Transl. no. 35 (1951). Reprint Amer. Math. Soc. Transl. (1) 8 (1962), 305-364.

[3] Charles Joiner, 'Free topological groups and dimesion', (to appear).

[4] A. G. Kurosh, Theory of Groups, Volume 1 (Chelsea Publishing Company, New York, 1960).

[5] Sidney A. Morris, 'Quotient groups of topological groups with no small subgroups', Proc. Amer. Math. Soc. 31 (1972), 625-626.

[6] Sidney A. Morris and H. B. Thompson, 'Free topological groups with no small subgroups', (to appear).

[7] Sidney A. Morris and H. B. Thompson, 'Invariant metrics on free topological groups', Bull. Austral. Math. Soc. 9 (1973), 83-88.

Flinders University

South Australia 